\title{
On the Radiation Properties of Split Ring Resonators (SRRs) at the Second Resonance
}

\author{
Simone Zuffanelli, Gerard Zamora, Member, IEEE, Pau Aguilà, Ferran Paredes, Ferran Martín, \\ Fellow, IEEE, and Jordi Bonache, Member, IEEE
}

\begin{abstract}
The radiation properties of split ring resonators (SRRs) at their second resonance frequency are studied for the first time in this work. In particular, the electric and magnetic dipole moments of the edge-coupled SRR (EC-SRR) are calculated analytically under the assumption of strong coupling between the internal and external rings. Based on these results, the radiation resistance and the radiation efficiency are obtained theoretically. Electromagnetic simulations of the structure reveal that there is very good agreement with the theoretical predictions, pointing out the validity of the proposed analysis. As a proof of concept, an SRR antenna prototype is designed and fabricated. Experimental data are in good agreement with the theoretical and simulated results, and demonstrate the validity of the SRR working at its second resonance frequency as a radiating element.
\end{abstract}

Index Terms - Split ring resonators (SRRs), planar antennas, radiation efficiency, metamaterials.

\section{INTRODUCTION}

Sal PLIT ring resonators (SRRs), first proposed by Pendry et al. [1] as an evolution of the structures proposed by Schelkunoff [2] and Hardy [3], have been widely used for the implementation of metamaterial-based or inspired microwave devices in the last years [4]. Due to their small electrical size and negative (and very high) magnetic polarizability above the fundamental resonance, SRRs can be used for the implementation of one-dimensional effective media metamaterials [5], including metamaterial transmission lines. Negative permeability (or mu-negative -MNG) [6], lefthanded (LH) [7] and composite right-/left-handed (CRLH) [8] transmission lines have been implemented by loading a host line with SRRs (and with other additional elements for LH and CRLH lines). These artificial lines and other artificial lines based on modifications of the SRR topology (including the complementary counterpart, i.e., the CSRR [9]) have been applied to improve the performance and/or the size of microwave components and to implement new functionalities.

This work has been supported by MINECO-Spain (projects TEC201017512 METATRANSFER, CONSOLIDER EMET CSD2008-00066, and TEC2013-40600-R COM-SEN-RFID), by FEDER funds, and by Generalitat de Catalunya (project 2014SGR-157). Ferran Martín has been awarded with an ICREA Academia Award.

The authors are with GEMMA/CIMITEC, Departament d'Enginyeria Electrònica, Universitat Autònoma de Barcelona, 08193 Bellaterra, Spain (email: Jordi.Bonache@uab.es).
Compact wideband and ultra-wideband (UWB) filters [10], power dividers [11] and microwave sensors [12]-[13] among other devices, have been implemented by means of SRR- or CSRR-loaded lines. The control of the phase constant and characteristic impedance (a unique feature of metamaterial transmission lines) in SRR- and CSRR-loaded lines has also been successfully applied to the design of leaky-wave antennas with broadside and backward-to-forward radiation capabilities [14]-[16]. Moreover, metamaterial structures and metamaterial-inspired resonators have been widely applied to electrically small antenna design, in order to reduce dimensions [17]-[18], obtain multiband and multi-frequency operation [19]-[21], high radiation efficiency [22], and achieve tuning capability [23]. In many of the works mentioned above, SRRs were used in order to achieve the improvements in the antenna response.

The resonance modes of SRRs have been extensively studied in [24], and the values of their resonance frequencies were analytically predicted in [25]. Nevertheless, since the particle is normally designed to work around its first (fundamental) resonance, all the theoretical studies have been focused on the properties of the SRR at that frequency. For instance, the first-order terms of the polarizability tensor of the particle have been quantified in [4],[26],[27],[28] by using the quasi-static analysis at the fundamental resonance. From such analysis, it follows that the SRR acts as a current loop at the fundamental resonance, thus suggesting the possibility of using it as a radiating element. However, since the particle size is in the order of 0.1 free-space wavelengths, and the radiation resistance of a current loop depends on the fourth power of the radius in terms of wavelengths, poor radiation efficiencies and narrow bandwidths are expected. For these reasons, the SRR has been rarely used as stand-alone radiating element at the fundamental resonance [29]. However, as it will be demonstrated in this work, the second resonance of the SRR exhibits interesting antenna properties in terms of radiation resistance and efficiency.

In this paper, an analytical study of the radiation resistance associated to the second resonance of the SRR is carried out, providing equations that can be used in the design process to obtain high radiation efficiency and good impedance matching. Given that the radius of the particle at the second resonance is typically in the order of 0.1 free-space wavelength (although it is electrically larger than the radius at the first resonance), the radiator can be treated as an electrically small antenna, according to commonly given definition $(k r<1$ [30], where $k$ 
is the free-space wavenumber and $r$ is the radius of the smallest sphere enclosing the antenna), thus simplifying the analysis of the problem. Due to its uniplanar geometry, the edge-coupled SRR (EC-SRR) is the topology considered throughout this work.

The paper is organized as follows. Section II is dedicated to the analysis of the particle. The electric and magnetic dipole moments and the radiation resistance of the SRR at the second resonance are predicted in Subsection II.A. The losses introduced by the conductors are then evaluated in Subsection II.B, and an expression for the radiation efficiency is presented in Subsection II.C, along with a discussion over the far-field properties of the particle. The theoretical expressions are compared to the electromagnetic simulations in Section III, where the radiation quality factor of the particle is also evaluated and compared to the theoretical limitations. In order to validate the theoretical and simulated results, an SRR antenna prototype working at the $900 \mathrm{MHz}$ ISM band (902$928 \mathrm{MHz}$ ) is designed, fabricated and subjected to experimental measurements, and the results are presented in Section IV. Finally, the main conclusions are highlighted in Section V.

\section{ANALYSIS}

\section{A. Radiation resistance}

The topology of the EC-SRR is shown in Fig. 1. The mean radii of the external and internal rings are $r_{A}$ and $r_{B}$, respectively. The ring trace width, $c$, is assumed to be the same for both rings, a condition which is usually complied in most of the designs involving the EC-SRR. The width of the cuts in each ring is not critical for the SRR response, since the capacitance associated to the cut can be neglected [4]. Its value is set greater than the distance between rings $d$ and, obviously, much smaller than the mean ring radius $r_{0}=\left(r_{A}+r_{B}\right) / 2$.

Provided that the particle is electrically small, its radiation is mainly produced by the first order (dipolar) terms of the electric and magnetic moments generated by the electric currents in the rings. The electric current distribution at the second resonance approximately satisfies $\boldsymbol{i}_{A}(\varphi)=-\boldsymbol{i}_{B}(\varphi \pm \pi)$ [24], where $\boldsymbol{i}_{A}$ and $\boldsymbol{i}_{B}$ is the current in the external (A) and internal (B) ring, respectively, and $\varphi$ is the angular position with respect to the $x$-axis. This relation is valid if the distance between rings, $d$, is small as compared to the mean ring radius $r_{0}$ (strong coupling condition).

Let us now focus on the radiation associated to the electric dipole moment, which can be calculated by evaluating the charge distribution along the rings. To this end, let us first describe the current profile along each ring. Since the length of each ring is approximately half-wavelength, the current along the ring circumference is assumed to be sinusoidal with a maximum value at the center ( $\varphi=0$ for ring $\mathrm{A}$ and $\varphi= \pm \pi$ for ring $\mathrm{B}$ ), and null at the ring edges (approximated to $\varphi= \pm \pi$ for ring $\mathrm{A}$ and $\varphi=0$ for ring $\mathrm{B}$ ). However, as it is corroborated later (see Fig. 2b), the current in each ring

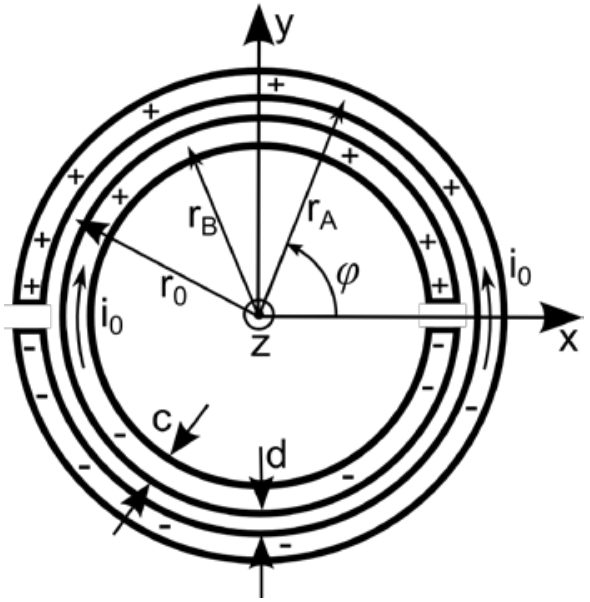

Fig. 1. Topology and charge and current distributions in the EC-SRR at the second resonance.

actually exhibits a slight discrepancy to this approximation, mainly because the length of each ring at the second SRR resonance is somewhat greater than the half-wavelength at the working frequency. Nevertheless, by means of this sinusoidal approximation to the rings currents, analytical expressions for the different variables of interest in this paper can be obtained, and the agreement between simulation/experiment and theory is good. The current in the external ring, of mean radius $r_{A}$, can thus be written as:

$$
\boldsymbol{i}_{A}(\varphi, t)=i_{0} \cos \left(\frac{\varphi}{2}\right) \cos \left(\omega_{0} t\right) \hat{\boldsymbol{\varphi}}
$$

where $\omega_{0}$ is the angular frequency and $i_{0}$ is the maximum current amplitude. By solving the well-known electric charge continuity equation, the linear charge density in the ring is found to be:

$$
\lambda_{A}(\varphi, t)=\frac{i_{0} \sin \left(\frac{\varphi}{2}\right) \sin \left(\omega_{0} t\right)}{2 r_{A} \omega_{0}} .
$$

Since (2) is odd with respect to the position angle $\varphi$, the total electric dipole moment associated to one ring is the sum of the infinitesimal dipole moments directed along the $\hat{\boldsymbol{y}}$ direction, namely:

$$
\boldsymbol{p}_{A}=\int_{0}^{\pi} \lambda_{A}(\varphi, t) 2 r_{A}^{2} \sin \varphi d \varphi \hat{\boldsymbol{y}}
$$

Integration of (3) gives:

$$
\boldsymbol{p}_{A}=\frac{4}{3} \frac{r_{A}}{\omega_{0}} i_{0} \sin \left(\omega_{0} t\right) \hat{\boldsymbol{y}}
$$

Since the internal ring $\mathrm{B}$, of mean radius $r_{B}$, exhibits an electric dipole moment with the same orientation and phase, the total dipole moment is the sum of the internal and external dipole moments, i.e.,

$$
\boldsymbol{p}=\frac{8}{3} \frac{r_{0}}{\omega_{0}} i_{0} \sin \left(\omega_{0} t\right) \hat{\boldsymbol{y}}
$$

where it has been assumed that the current distributions in rings A and B satisfy $\boldsymbol{i}_{A}(\varphi)=-\boldsymbol{i}_{B}(\varphi \pm \pi)$, as mentioned before. 
Since the radiating particle is electrically small, it is possible to consider the total moment given by (5) as an infinitesimal electric dipole moment located in the $y$-axis. Thus, by using the Larmor equation [31] for the radiated power by a given electric dipole moment of amplitude $p_{0}$, that is

$$
P_{\text {rad }}^{\mathrm{E}}=c_{0}^{2} \frac{Z_{0} k^{4} p_{0}^{2}}{12 \pi}
$$

the total radiation resistance referred to the maximum current $i_{0}$ can be expressed as follows:

$$
R_{\mathrm{rad}}^{\mathrm{E}}=\frac{128}{27} \pi Z_{0}\left(\frac{r_{0}}{\lambda_{0}}\right)^{2} \approx 5600\left(\frac{r_{0}}{\lambda_{0}}\right)^{2} \Omega .
$$

In (6) and (7), $c_{0}$ is the speed of light in free-space, $Z_{0}$ is the impedance of free-space, and $\lambda_{0}$ is the free-space wavelength at the second resonance frequency, $f_{0}$.

It is now interesting to compare the value found in (7) with the radiation resistance of a small dipole, which can be expressed by means of the well-known expression:

$$
R_{\mathrm{rad} 0}=20 \pi^{2}\left(\frac{l}{\lambda_{0}}\right)^{2} \approx 790\left(\frac{r}{\lambda_{0}}\right)^{2} \Omega
$$

where $r$ is the length of each dipole arm. As can be seen by comparing (7) and (8), for a given radiator size ( $r_{0}$ for the SRR or $r$ for the dipole), the SRR working at the second resonance exhibits a much higher radiation resistance as compared to the one of a small electric dipole. This can be explained arguing that the SRR takes advantage of most of the area enclosed by its lateral dimensions, while the dipole only distributes its charges along a line.

The magnetic dipole moment associated to the external ring can be calculated as

$$
\boldsymbol{m}_{A}=\frac{1}{2} \int_{-\pi}^{\pi} \hat{\boldsymbol{r}} \times \mathbf{i}_{A}(\varphi, t) r_{A}^{2} d \varphi
$$

providing the following expression:

$$
\boldsymbol{m}_{A}=2 r_{A}^{2} i_{0} \cos \left(\omega_{0} t\right) \hat{\mathbf{z}} .
$$

As explained above, the current in the internal ring flows in the opposite direction, and generates an opposite axial magnetic dipole moment. Therefore, the total magnetic dipole moment can be written as:

$$
\boldsymbol{m}=4 r_{0}\left(r_{A}-r_{B}\right) i_{0} \cos \left(\omega_{0} t\right) \hat{\mathbf{z}} .
$$

The radiation resistance associated to an electrically small magnetic dipole moment of amplitude $m_{0}$ can be found from the radiated power, given by [32]

$$
P_{\text {rad }}^{\mathrm{M}}=\frac{Z_{0} k^{4} m_{0}^{2}}{12 \pi} .
$$

Since $m_{0}$ is, in our case, equal to $4 r_{0}\left(r_{\mathrm{A}}-r_{\mathrm{B}}\right) i_{0}$, the radiation resistance is:

$$
R_{\mathrm{rad}}^{\mathrm{M}}=\frac{128}{3} \pi^{3} Z_{0}\left(\frac{r_{0}}{\lambda_{0}}\right)^{2}\left(\frac{c+d}{\lambda_{0}}\right)^{2} .
$$

It can be easily verified that, due to a nearly complete cancellation of the axial magnetic moments of the internal and external rings, the power radiated by the magnetic dipole is much smaller (two orders of magnitude) than the power associated to the electric dipole. Therefore, to a good approximation, the radiation resistance of the particle at second resonance is dominated by the one associated to its electric dipole moment, that is,

$$
R_{\mathrm{rad}} \approx R_{\mathrm{rad}}^{\mathrm{E}} .
$$

The potential of SRRs as radiating elements operating at their second resonance is demonstrated by evaluating (14) for typical values of $r_{0} / \lambda_{0}$, which are in the order of 0.1 (when no substrate is used, see Section III). The resulting radiation resistance values are very close to the radiation resistance of the commonly used, and very well-known, half-wave dipole antenna $(73 \Omega)$. This fact suggests that half-wave dipole antennas can be replaced with SRRs (operating at the second resonance) without the need of a matching network, thereby reducing the maximum dimension of the antenna (defined as the radius of the minimum sphere enclosing the antenna) by a factor 2.5, approximately.

\section{B. Loss resistance}

Let us now focus on the ohmic losses introduced by the metallic layer of the SRR, which are normally the main loss mechanism at microwave frequencies [33]. As for the electric dipole moment calculation, the current distribution along the ring perimeter is taken sinusoidal, with a null at the edges. The current density distribution $\boldsymbol{J}$ within the ring cross section decays exponentially from the surface, with a penetration depth (skin depth) depending on the working frequency $f_{0}$. Since the conductor thickness $h$ is usually much smaller than its width $c$, only the top and bottom faces are considered to carry current, neglecting the small contribution from the lateral faces.

The assumptions above lead to the following expression for $\boldsymbol{J}_{A}$ (the current density in the outer ring) in phasorial form:

$$
\boldsymbol{J}_{A}(r, \varphi, z)=\left\{\begin{array}{lc}
J_{0}(r) e^{-\gamma\left(z+\frac{h}{2}\right)} \cos \left(\frac{\varphi}{2}\right) \hat{\boldsymbol{\varphi}} & -\frac{h}{2}<z<0 \\
J_{0}(r) e^{\gamma\left(z-\frac{h}{2}\right)} \cos \left(\frac{\varphi}{2}\right) \hat{\boldsymbol{\varphi}} & 0<z<\frac{h}{2}
\end{array}\right.
$$

where $\gamma=(1+j) / \delta$ is the complex propagation constant inside the conductor and $\delta$ is the skin depth, given by $\delta=(2 / \omega \mu \sigma)^{1 / 2}$ [33], where $\mu$ and $\sigma$ are the permeability and conductivity of the conductor material, respectively. The above expressions (for $\gamma$ and $\delta$ ) are valid for low-loss conductors, i.e., for those satisfying $\sigma \gg \omega \varepsilon$ ( $\varepsilon$ being the permittivity). The radial dependence of $\boldsymbol{J}_{A}$ [i.e., $\boldsymbol{J}_{0}(r)$ ] is cumbersome to be expressed analytically. Indeed, due to the proximity effect [34] between the internal and external rings of the SRR, the current tends to accumulate in the regions of the upper and lower sides of the rings closer to the slot (of width $d$ ) between them. Given the position of the current zeroes and maxima in the rings, one can deduce that the proximity effect varies with the angle $\varphi$, being 
maximum for $\varphi=\pi$ and minimum for $\varphi=0$ for the external ring (and vice versa for the internal ring). We have modeled the overall influence of the proximity effect on the ohmic losses by means of an effective width $c_{\text {eff }}$, approximated by $c / 2$, where the current density is assumed to be constant with $r$. This approximation is based on the assumption that the current for the external ring is uniformly distributed in the radial direction when $\varphi=0$, and concentrated in the extreme adjacent to the slot when $\varphi \rightarrow \pm \pi$, and that a linear variation with the angle between these two extreme situations arises (note that for the internal ring the current is uniformly distributed in the radial direction when $\varphi= \pm \pi$, and concentrated in the extreme adjacent to the slot when $\varphi \rightarrow 0$ ). Therefore, $J_{0}(r)$ is assumed to be constant with $r\left[J_{0}(r)=J_{0}\right]$ for radial values between $r_{A}-c_{\text {eff }} / 2$ and $r_{A}+c_{\text {eff }} / 2$, and null elsewhere.

The power loss in the outer ring, inferred from Ohm's law, is

$$
P_{\text {loss, } \mathrm{A}}=\frac{1}{2} \int_{-\pi}^{\pi} \int_{\left(-\frac{h}{2}\right)}^{\left(\frac{h}{2}\right)} \int_{\left(r_{A}-\frac{c_{\text {eff }}}{2}\right)}^{\left(r_{A}+\frac{c_{\text {eff }}}{2}\right)} \frac{\left|J_{A}(r, \varphi, z)\right|^{2}}{\sigma} r d r d z d \varphi .
$$

By solving (16), the power loss is found to be

$$
P_{\text {loss }, \mathrm{A}}=\frac{\pi c_{\mathrm{eff}} r_{A} \delta\left(1-e^{-\frac{h}{\delta}}\right)}{2 \sigma} J_{0}^{2} .
$$

The power loss in the inner ring can be obtained by using the same procedure. Thus, the total power loss can be written as:

$$
P_{\text {loss }}=\frac{\pi c_{\text {eff }} r_{0} \delta\left(1-e^{-\frac{h}{\delta}}\right)}{\sigma} J_{0}^{2} .
$$

Let us now compute the total current $i_{0}$ flowing at the input section $(\varphi=0)$, in order to calculate the loss resistance. By integrating the current density over the effective crosssectional area, namely

$$
i_{0}=\int_{\left(-\frac{h}{2}\right)}^{\left(\frac{h}{2}\right)} \int_{\left(r_{A}-\frac{c_{\text {eff }}}{2}\right)}^{\left(r_{A}+\frac{c_{\text {eff }}}{2}\right)}\left(J_{A}(r, 0, z) \cdot \hat{\boldsymbol{\varphi}}\right) d r d z
$$

it can be found that the squared RMS (root mean square) current can be expressed as:

$$
\frac{\left|i_{0}\right|^{2}}{2}=J_{0}^{2} c_{\text {eff }}^{2} \delta^{2}\left[1+\mathrm{e}^{-\frac{h}{\delta}}-2 \mathrm{e}^{-\frac{h}{2 \delta}} \cos \left(\frac{h}{2 \delta}\right)\right] .
$$

Finally, the loss resistance is obtained by dividing the power loss (18) by the squared RMS current (20), obtaining

$$
R_{\text {loss }}=\frac{\pi r_{0}}{\sigma c_{\text {eff }} \delta}\left[\operatorname{coth}\left(\frac{h}{2 \delta}\right)-\operatorname{csch}\left(\frac{h}{2 \delta}\right) \cos \left(\frac{h}{2 \delta}\right)\right]^{-1} .
$$

The hyperbolic function on the right side of (21) can be approximated by the hyperbolic cotangent with the same argument, that is

$$
\left[\operatorname{coth}\left(\frac{h}{2 \delta}\right)-\operatorname{csch}\left(\frac{h}{2 \delta}\right) \cos \left(\frac{h}{2 \delta}\right)\right]^{-1} \approx \operatorname{coth}\left(\frac{h}{2 \delta}\right)
$$

with a maximum error of $20 \%$ for $h=3.7 \delta$. We will later discuss the error introduced by (22) on the radiation efficiency, which is the parameter of interest in the present analysis.

By combining (21) and (22), the loss resistance can be expressed in a very simple form, that is:

$$
\mathrm{R}_{\text {loss }} \approx \frac{2 \pi r_{0}}{\sigma c \delta} \operatorname{coth}\left(\frac{h}{2 \delta}\right)
$$

where the effective width has been approximated by $c_{\text {eff }} \approx c / 2$, as mentioned above.

\section{Antenna properties}

Let us now discuss the far-field properties of the particle. The radiation efficiency of any antenna is defined as the ratio between the radiated power and the accepted power. This magnitude can be expressed as a function of the ratio between the loss resistance (23) and the radiation resistance (14), that is $\eta_{\text {rad }}=\left(1+R_{\text {loss }} / R_{\text {rad }}\right)^{-1}$, obtaining:

$$
\eta_{\text {rad }} \approx\left[1+\frac{27}{64} c_{0} \sqrt{\frac{\pi}{\mu_{0}}}\left(c r_{0} \sqrt{f_{0}^{3} \sigma}\right)^{-1} \operatorname{coth}\left(\frac{h}{2 \delta}\right)\right]^{-1}
$$

where $\mu_{0}$ is the permeability of free space. It is worth mentioning that, for reasonable combinations of thickness, width and conductivity, the maximum error $(20 \%$ for $h=3.7 \delta$ ) introduced in (22) is greatly reduced in the final expression (24) of the radiation efficiency [e.g., $4 \%$ for $h=35 \mu \mathrm{m}, c=0.2 \mathrm{~mm}$ and $\sigma=5 \cdot 10^{6} \mathrm{~S} / \mathrm{m}$, which is the worst case in our analysis (see Section III)].

The directivity of the radiated fields, according to the assumptions made above, should be the same as the one of an elemental electric dipole (1.76 dB). However, due to the nonzero length of the particle in the $x$ direction, a slightly bidirectional pattern is expected over the $H$-plane $\left(\varphi=0^{\circ}\right)$, thus resulting in a higher value of the directivity.

The cross-polar component of the radiated fields is mainly associated to the radiation produced by the magnetic dipole moment, which was evaluated in section IIA. In fact, as can be deduced by comparing (5) and (11), a $90^{\circ}$ phase-shift exists between the electric and magnetic dipole moments. Hence, in the directions which provide orthogonality between the fields radiated by the magnetic and the electric moments, i.e. the $E$ plane, the radiation of the magnetic moment is purely crosspolar. Since the magnetic dipole moment is oriented along the $z$-axis, its far-field contribution is maximized for $\theta=90^{\circ}$. Hence, in this direction the value of the cross-polarization level is maximized, and can be evaluated dividing (13) by (7), obtaining:

$$
X P O L_{\max }=9 \pi^{2}\left(\frac{c+d}{\lambda_{0}}\right)^{2}
$$


(a)

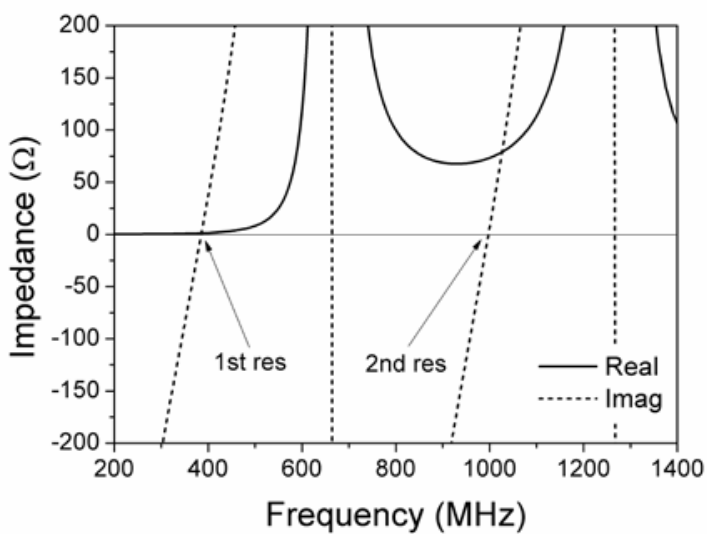

(b)

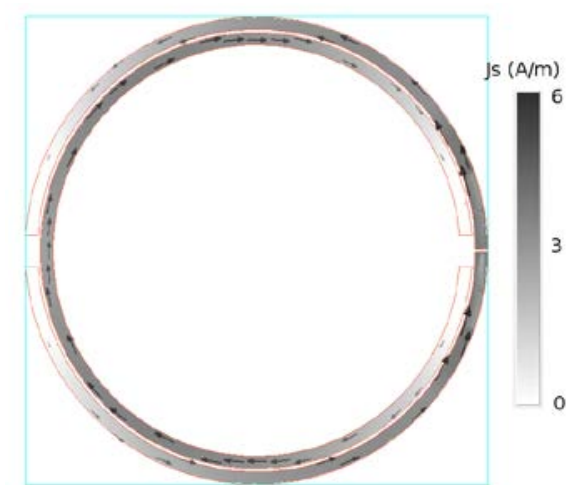

Fig. 2. (a) Input impedance and (b) electric current distribution at the second resonant frequency $(1 \mathrm{GHz})$ of the simulated lossless SRR (no dielectric substrate is considered).

\section{Simulation Results}

In order to validate the analytical results presented above, an EC-SRR has been simulated by means of the commercial software Agilent Advanced Design System (ADS). The geometry of the particle has been adjusted to locate the second resonance at the frequency $f_{0}=1 \mathrm{GHz}\left(\lambda_{0}=300 \mathrm{~mm}\right)$ when no substrate is used. The values for the geometric parameters are $r_{A}=36.5 \mathrm{~mm}, \quad r_{B}=34 \mathrm{~mm} \quad c=2 \mathrm{~mm}, \quad d=0.5 \mathrm{~mm}$, $r_{0}=35.25 \mathrm{~mm}\left(0.118 \lambda_{0}\right)$, and the cut width is set to $5 \mathrm{~mm}$.

The discrete port is placed across a $0.4 \mathrm{~mm}$ gap opened at the center of the external ring $\left(\varphi=0^{\circ}\right)$, where a current maximum $i_{0}$ is expected to occur. Therefore, in a lossless case, the input resistance $R_{\text {in }}$ of the system corresponds to the radiation resistance $R_{\text {rad }}$ of the particle. Hence, in the first set of simulations the metal is treated as a perfect conductor, in order to isolate the radiation resistance of the SRR and to verify (14).

The simulated input impedance of the SRR (without dielectric substrate), depicted in Fig. 2(a), clearly reveals the first $(0.4 \mathrm{GHz})$ and second $(1 \mathrm{GHz})$ resonant frequencies of the SRR, and the first anti-resonant frequency $(0.66 \mathrm{GHz})$, which corresponds to the intrinsic resonance of the inner ring. Note also that the radiation resistance at the second resonance $(73 \Omega)$ is nearly two orders of magnitude greater than the radiation resistance at the first resonance $(1 \Omega)$, and is exactly equal to the radiation resistance of a canonical half-wave dipole. The simulated electric current density distribution at

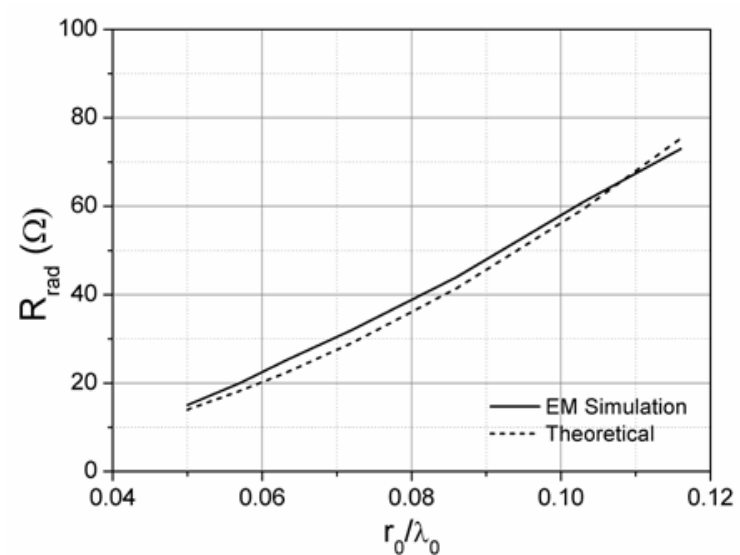

Fig. 3. Radiation resistance at second resonance as a function of the SRR mean radius relative to the wavelength. Note that the radiation resistance is roughly $73 \Omega$ (i.e., the radiation resistance value of a half-wave dipole) for $r_{0} / \lambda_{0}=0.118$

the second resonance (Fig. 2(b)) is in good agreement with the approximated theoretical distribution described in Section II.A, i.e., the current in the internal and external rings flow in opposite directions, and their amplitudes are similar. However, since the length of each ring is slightly greater than halfwavelength, the maximum at the center of the rings splits into two maxima symmetrically positioned around the center. Nevertheless, expression (1) is a useful approximation, as it allows simplifying the analytical treatment maintaining high accuracy in the results.

In order to validate (14), a lossless dielectric of thickness $h_{s}=2.54 \mathrm{~mm}$ is then added as substrate, allowing to control the value of the second resonance frequency $f_{0}$, and consequently the value of $\lambda_{0}$. By increasing the dielectric permittivity $\varepsilon_{r}$ of the substrate, the value of $r_{0} / \lambda_{0}$ is gradually decreased, and the simulated input resistance of the particle is compared in each case to the radiation resistance calculated by (14). The results, depicted in Fig. 3, reveal that there is very good agreement between theory and simulation.

It is worth to mention that, in order to modulate the electrical size of the particle without changing the substrate dielectric constant, it is also possible to vary the coupling between rings, mainly controlled by the slot width $d$. In fact, according to the theory of coupled resonators, decreasing the coupling (while fixing $r_{0}$ ) reduces the frequency split between first and second resonance. Therefore, the second resonance is lowered and the particle becomes electrically smaller, providing an additional degree of freedom at the design stage. However, only relatively small changes of $r_{0} / \lambda_{0}$ are possible by using this technique. Moreover, if the coupling between rings is very small, the assumptions for the derivation of equations (5) and (11) no longer hold. The reason is that the difference between the internal and external rings radii increases with the distance $d$, and the internal and external current amplitudes diverge as well.

A second set of simulations, without substrate, takes into account the ohmic losses in the metallic conductor, in order to validate (24). The simulated radiation efficiency as a function 

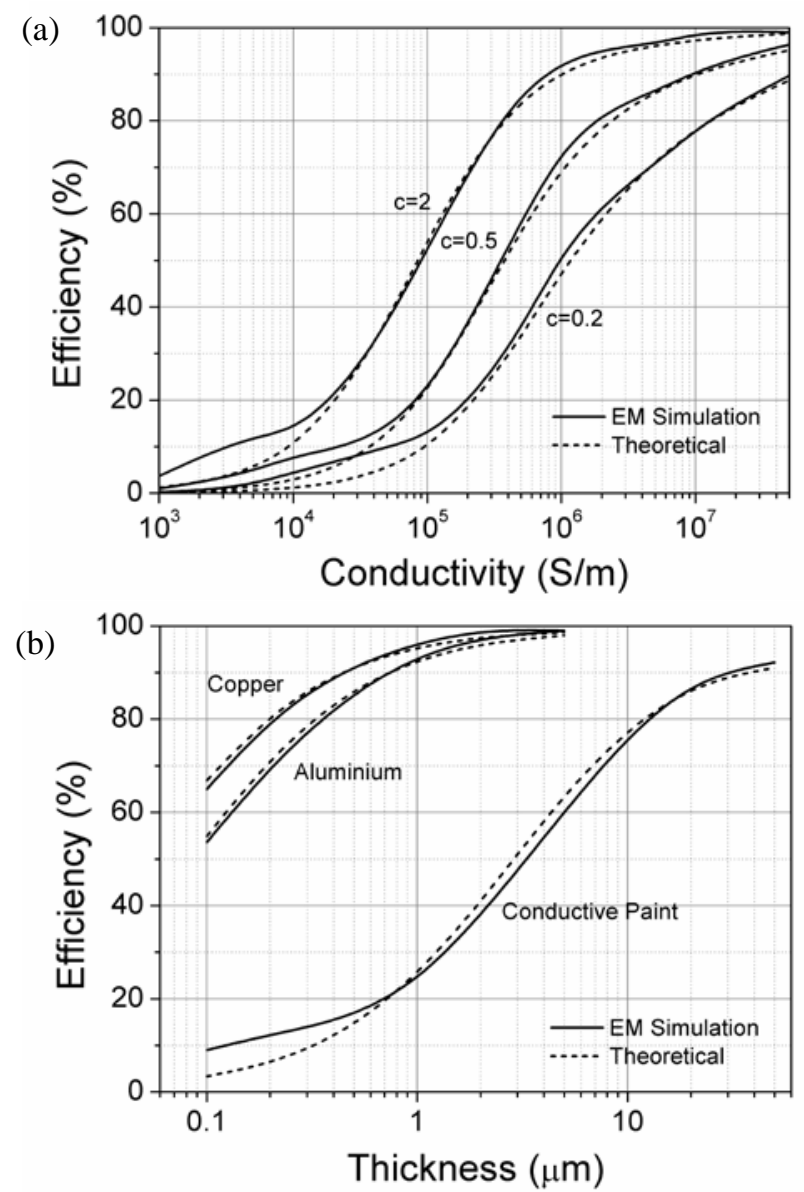

Fig. 4. Simulated and theoretical radiation efficiency (a) versus conductivity for $h=35 \mathrm{um}$ and (b) versus conductor thickness for $c=2 \mathrm{~mm}$. The considered conductive paint has a conductivity $\sigma=10^{6} \mathrm{~S} / \mathrm{m}$ [35]-[36].

of the metal conductivity for different values of the strip width $c$, setting the value of the metal thickness to $h=35 \mu \mathrm{m}$, is shown in Fig. 4(a). The thickness dependence of the radiation efficiency for a strip width of $c=2 \mathrm{~mm}$ is simulated and depicted in Fig. 4(b) for different materials. Very good agreement between electromagnetic simulations and theoretical predictions is observed for efficiency values greater than $15 \%$, which is the region of interest in practical antennas.

Let us now describe the simulated radiation pattern at the frequency of $1 \mathrm{GHz}$ (no substrate used), depicted in Fig. 5, which was obtained by means of the ADS Momentum 2-D radiation pattern. As can be seen, the radiation pattern on the E-plane $\left(\varphi=90^{\circ}\right)$ corresponds, as expected, to the radiation from an electric dipole oriented towards the $y$-axis. However, the value of the directivity is $D_{0}=2.7 \mathrm{dBi}$, which is $1 \mathrm{~dB}$ higher than the elemental dipole due to the array effect associated to the finite dimension of the SRR in the $x$ direction, as mentioned in section II.C. As expected, the crosspolarization levels are low $(X P O L<-21 \mathrm{~dB})$ and in very good agreement with (25), which provides $X P O L_{\max }=-22 \mathrm{~dB}$. Therefore, the radiated fields can be considered linear on most of the radiating sphere.

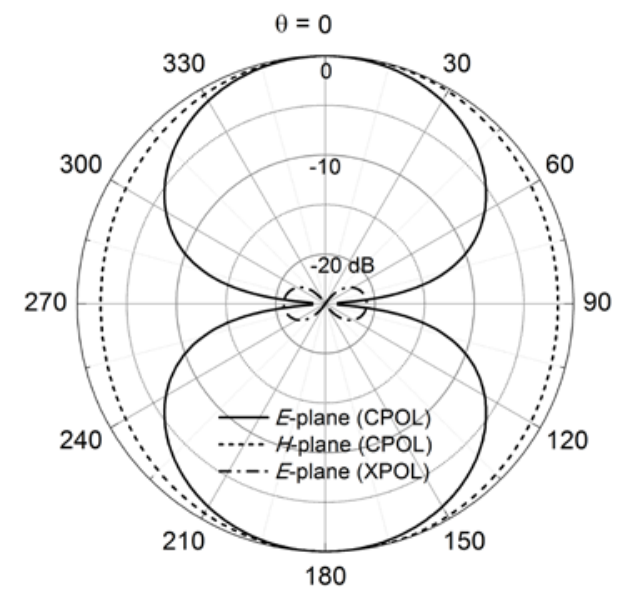

Fig. 5. Simulated normalized radiation pattern of the SRR at $f_{0}=1 \mathrm{GHz}$ in the $E$-plane $\left(\varphi=90^{\circ}\right)$ and $H$-plane $\left(\varphi=0^{\circ}\right)$.

As a final consideration, let us estimate the radiation quality factor $Q_{\text {rad }}$ of the antenna at the second resonance, based on the simulated input impedance in the lossless case. To do this, we use the approximated expression derived in [37]:

$$
Q_{\mathrm{rad}} \approx \frac{\omega_{0}\left|Z^{\prime}\left(\omega_{0}\right)\right|}{2 R\left(\omega_{0}\right)}
$$

where $Z^{\prime}\left(\omega_{0}\right)$ is the first derivative of the input impedance with the angular frequency and $R\left(\omega_{0}\right)$ is the input resistance of the antenna, both evaluated at $\omega_{0}=2 \pi f_{0}$. By using (26), the value of the quality factor was found to be $Q_{\text {rad }} \approx 17$, leading to a half-power fractional bandwidth $F B W \approx 2 / Q_{\text {rad }} \approx 0.12$ [37] (considering conjugate matching). As expected, due to the planar geometry of the particle, the quality factor is larger than the minimum value for the quality factor of an electrically small antenna, as predicted by Chu in [38]-[39]. In fact, according to such a limitation, the minimum $Q_{\text {rad }}$ for an antenna enclosed in a sphere of radius $r \approx 0.12 \lambda_{0}$ is $(k r)^{-3}=2.3$. Nevertheless, it was shown in [40] that, according to the Gustafsson limit for small planar antennas enclosed in a circle of radius $r$, the lower $Q_{\text {rad }}$ bound increases by a factor $9 \pi / 8 \approx 3.5$ (considering directivity 1.5 ) with respect to the general case of a non-planar antenna (where the lower bound for the radiation quality factor is calculated by using the Chu formula). This provides a lower bound of $Q_{\mathrm{rad}}=8$, which is still smaller than the value found in our case. However, the radiation quality factor of the SRR antenna is similar to that of a small planar rectangular dipole antenna (with a length-towidth ratio around unity), and much better as compared to small thin dipole antennas [40]-[41].

\section{PROTOTYPE DESIGN AND MEASUREMENTS}

In this section, the theoretical analysis presented in Section II is applied to the design of an SRR antenna prototype, in order to validate its usefulness in the design stage, and to confirm the radiation properties of the SRR antenna introduced in this work. As a practical application example, the prototype was 


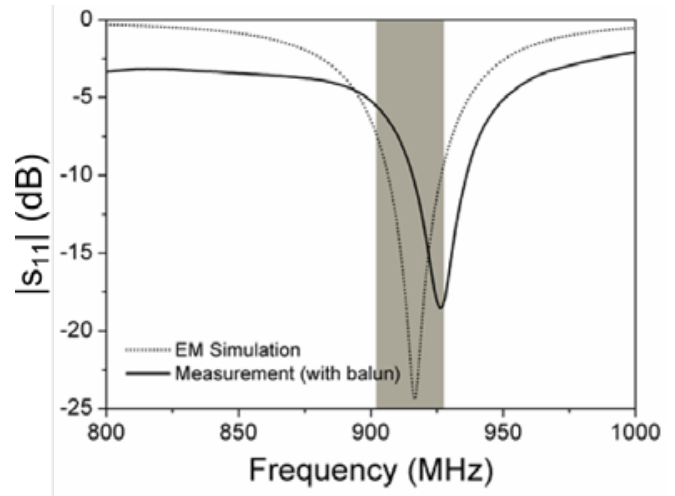

Fig. 6. Simulated and measured power reflection coefficient of the SRR antenna prototype. The $900 \mathrm{MHz}$ ISM band is depicted in gray.

designed to operate at the center of the $900 \mathrm{MHz}$ ISM band (902-928 MHz), i.e., at the frequency $f_{0}=915 \mathrm{MHz}$, matched to a $50 \Omega$ port.

\section{A. Design process and simulation results}

The first step in the design process is to determine the SRR mean radius $r_{0}$, in order to adjust the antenna input resistance to the required value of $R_{0}=50 \Omega$. In a general case, since the antenna input resistance at resonance is $R_{\text {in }}=R_{\text {rad }}+R_{\text {loss }}$, i.e. it is obtained by summing (7) and (23), its radius dependence is a second order polynomial of the form $R_{\mathrm{in}}=a r_{0}^{2}+b r_{0}$, where $a$ and $b$ depend on the frequency $f_{0}$, the metal conductivity $\sigma$ and the geometrical parameters $c$ and $h$. As a result, the required mean radius $r_{0}$ is obtained by solving a second order equation (which provides a unique positive solution) after the parameters mentioned above $\left(f_{0}, \sigma, c, h\right)$ have been set to a given value. However, in case the radiation efficiency is expected to be sufficiently high (see Fig. 4), the loss resistance can be neglected, and by using (7) the required mean radius can be approximated to

$$
r_{0} \approx 4 \cdot 10^{6} f_{0}^{-1} \sqrt{R_{0}}
$$

where all the quantities are expressed in SI units. Since the metal used for the prototype is copper with a thickness of $h=35 \mu \mathrm{m}$, setting the strip width to $c=2 \mathrm{~mm}$ allows using (27), because the expected efficiency is 98\% (neglecting the substrate). The result is $r_{0}=31 \mathrm{~mm}$.

The second and final step in the design stage is to adjust the second resonance of the SRR (with radius $r_{0}$ ) at the desired frequency $f_{0}$. Firstly, a dielectric substrate with the proper combination of relative permittivity $\varepsilon_{r}$ and thickness $h_{s}$ is chosen, obtaining a resonant frequency near to the desired value. Finally, by tailoring the distance $d$ between rings, a fine adjustment of the frequency of resonance is done. In our case, we used a Rogers $R 04003$ substrate $\left(\varepsilon_{r}=3.55, h_{s}=0.81 \mathrm{~mm}\right.$, $\tan \delta=0.0027$ ) and the ring distance was found to be $d=1.6 \mathrm{~mm}$. The ring cut width and the port gap, which values are not relevant to the SRR response, were set to $5 \mathrm{~mm}$ and $0.8 \mathrm{~mm}$, respectively.

The simulated power reflection coefficient of the SRR antenna prototype is depicted in Fig. 6. As expected, good impedance matching is achieved at the working frequency

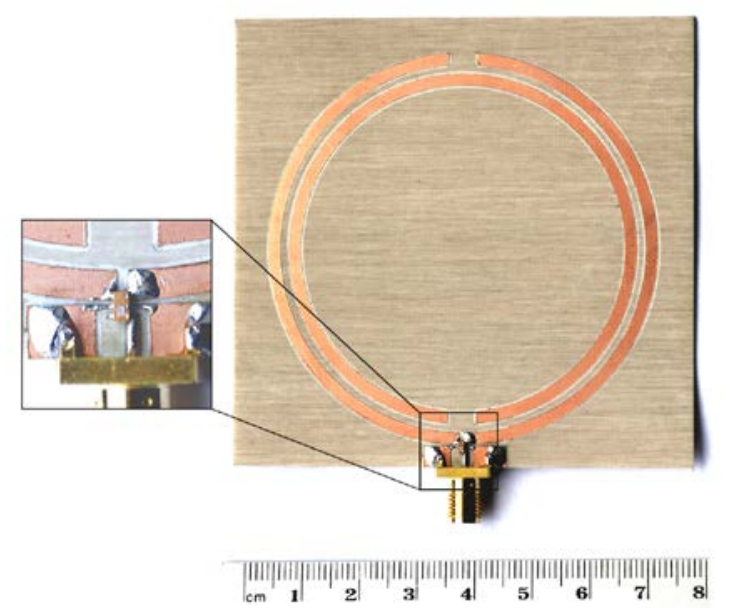

Fig. 7. Fabricated SRR antenna prototype. The SMD-packaged balun can be seen at the center of the close-up.

$f_{0}=915 \mathrm{MHz}$. The simulated radiation efficiency and antenna gain are $\eta_{\text {rad }}=92 \%$ (lower to the predicted $98 \%$ due to the presence of the dielectric losses) and $G_{0}=2.2 \mathrm{~dB}$.

\section{B. Fabrication and experimental results}

To validate the simulated results, the layout described in Section IV-A was fabricated (Fig. 7) by means of a PCB drilling machine ( $L P K F-H 100)$. The layout was provided with soldering pads, in order to mount a SMD-packaged high frequency ceramic balun (Johanson Technology 0900BL15C050 [42]) across the SRR port gap, and a SMA connector to feed the balun and the antenna. The substrate was cut square-shaped with a side length of $80 \mathrm{~mm}$.

The power reflection coefficient of the system was measured by means of an Agilent N5221A network analyzer, and the results (Fig. 6) are in good agreement with simulations, showing a small frequency shift $(10 \mathrm{MHz})$ and a good impedance matching level $(-18.5 \mathrm{~dB})$. However, since the presence of the balun was not taken into account in the simulations, some differences between simulation and measurement arise. In particular, the measured notch depth and out-of-band response are limited by the return and insertion losses of the balun, respectively.

The antenna gain was evaluated by using the following method (the measurement setup is outlined in Fig. 8a). The prototype was placed inside a Wavecontrol WaveCell TEM cell, and oriented towards the direction of the maximum radiation $\left(\theta=0^{\circ}, \varphi=90^{\circ}\right)$. The cell was excited with a pure tone at the frequency of $925 \mathrm{MHz}$, generated by means of an Agilent N5182A vector signal generator and amplified through a RFPA RF101000-10 solid state RF amplifier. The signal received by the antenna was delivered, by means of a coaxial cable, to the input port of an Agilent N9020A signal analyzer, where the input power $P_{r}$ was measured. Finally, the antenna was removed from the chamber, and replaced by an electric field probe (Wavecontrol EFCube) placed at the same position. The electric field strength $E_{\mathrm{RMS}}$ measured by the probe was used to evaluate the antenna gain $G_{0}$ as follows: 
(a)

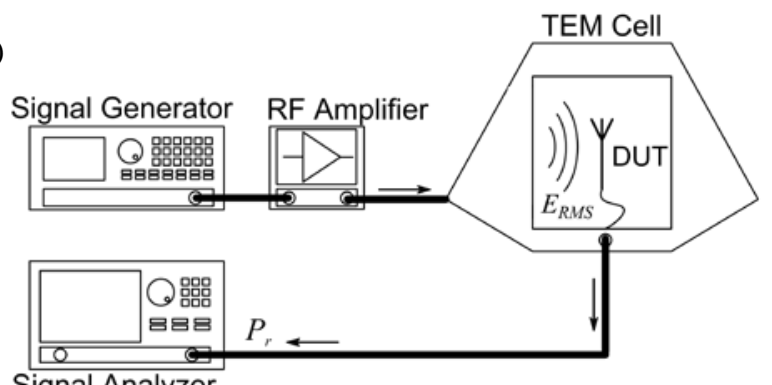

(b)

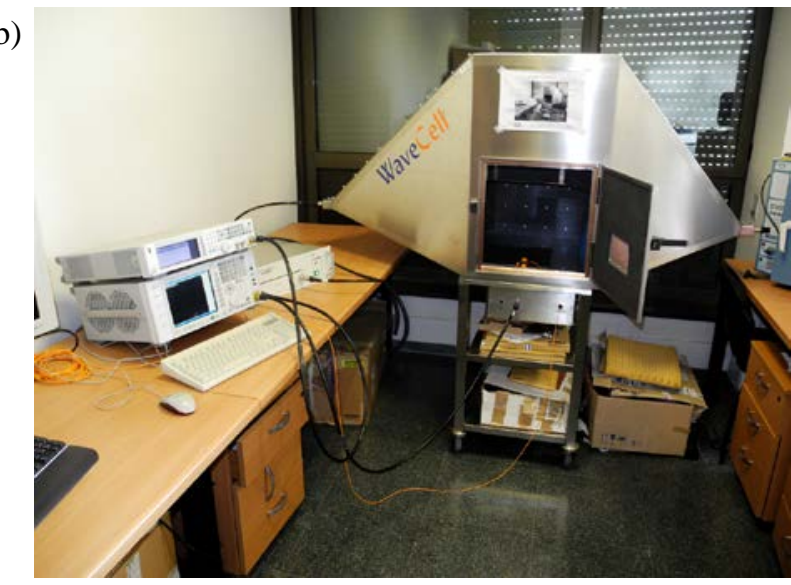

Fig. 8. (a) Scheme and (b) photograph of the antenna gain measurement setup.

$$
G_{0}=\frac{4 \pi Z_{0}}{\lambda_{0}^{2}} \frac{P_{r} \cdot \alpha}{E_{\mathrm{RMS}}^{2}}
$$

where the dimensionless parameter $\alpha(2.1 \mathrm{~dB})$ accounts for the losses introduced by the cable $(0.9 \mathrm{~dB})$ and the bal un $(1.2 \mathrm{~dB}$ [42]). Equation (28) is based on the common definition of antenna effective area as the relation between the received power and the incident power density, and assumes a pure TEM propagation inside the cell. By using (28), the experimental antenna gain was evaluated to be $G_{0}=2.05 \mathrm{~dB}$, which is very close to the predicted value (2.2 dB, Sec. IV-A).

\section{CONCLUSION}

The radiation properties of the EC-SRR at the second resonance, in terms of radiation resistance, efficiency, directivity and polarization, have been studied in this work. Starting from the calculation of the electric and magnetic dipole moments of the particle, it has been found that the main radiation mechanism is associated to the electric dipole moment generated along the plane of the particle, which results from the constructive contributions of the internal and external rings. Based on this analysis, an expression for both the radiation resistance and the radiation efficiency under the hypothesis of strong coupling between the rings has been presented. To validate the analysis, full-wave simulations of the radiation resistance and efficiency have been carried out, and very good agreement with the predicted theoretical values for different SRR parameters (i.e., conductivity, thickness and width of the metallic strips) has been found. As a proof of concept, a prototype of the SRR antenna has been designed and fabricated to work at the $900 \mathrm{MHz}$ ISM band, and the experimental data validated both the theoretical and simulated results in terms of impedance matching and antenna gain.

According to the study presented in this work, the SRR proves to be a valuable alternative to the commonly used halfwave dipole, especially when a reduction of the maximum antenna dimension is needed while maintaining a similar value of the radiation resistance. As a result, a SRR antenna operating at the second resonance can replace a half-wave dipole antenna without the need of any additional matching network. Moreover, its radiation pattern, which can provide a gain of $2.7 \mathrm{~dB}$, i.e., $0.5 \mathrm{~dB}$ higher than the one of the halfwave dipole, can be preferred in some applications where a more directional pattern is needed.

\section{REFERENCES}

[1] J. B. Pendry, A. J. Holden, D. J. Robbins, and W. J. Stewart, "Magnetism from conductors and enhanced nonlinear phenomena," IEEE Trans. Microw. Theory Techn., vol. 47, no. 11, pp. 2075-2084, Nov. 1999.

[2] S. Schelkunoff and H. Friis, Antennas: Theory and practice, John Wiley and Sons, 1952.

[3] W. Hardy and L. Whitehead, "Split-ring resonator for use in magnetic resonance from 200-2000 MHz,” AIP Rev. Sci. Instrum., vol. 52, no. 2, pp.213-216, 1981.

[4] R. Marques, F. Martin, and M. Sorolla, Metamaterials with Negative Parameters: Theory, Design and Microwave Applications, John Wiley and Sons, 2008.

[5] R. Marqués, J. Martel, F. Mesa, and F. Medina, "Left-handed-media simulation and transmission of EM waves in subwavelength split-ringresonator-loaded metallic waveguides," Phys. Rev. Lett., vol. 89, p. 183901, 2002.

[6] F. Falcone, F. Martin, R. Marqués, J. Martel, J. Bonache, T. Lopetegi, M. A. Laso, and M. Sorolla, "Implementation of negative $\mu$ medium in coplanar waveguide technology,” Proc. SPIE 5445, Microwave and Optical Technology 2003, p. 192, Apr. 7, 2004.

[7] F. Martin, J. Bonache, F. Falcone, M. Sorolla, and R. Marques, "Split ring resonator-based left-handed coplanar waveguide," Appl. Phys. Lett., vol. 83, pp. 4652-4654, Dec. 2003.

[8] J. Selga, M. Gil, J. Bonache, and F. Martín, "Composite right/left handed coplanar waveguides loaded with split ring resonators and their application to high pass filters," IET Microw. Ant. Propag., vol. 4, no. 7, pp. 822-827, July 2010.

[9] F. Falcone, T. Lopetegi, J.D. Baena, R. Marqués, F. Martín, and M. Sorolla, "Effective negative- $\varepsilon$ stop-band microstrip lines based on complementary split ring resonators", IEEE Microw. Wireless Compon. Lett., vol. 14, no. 6, pp. 280-282, June 2004.

[10] M. Gil, J. Bonache, J. García-García, J. Martel, and F. Martín, "Composite Right/Left Handed (CRLH) Metamaterial Transmission Lines Based on Complementary Split Rings Resonators (CSRRs) and Their Applications to Very Wide Band and Compact Filter Design,” IEEE Trans. Microw. Theory Techn., vol. 55, no. 6, pp. 1296-1304, June 2007.

[11] M. Gil, J. Bonache, I. Gil, J. Garcia-Garcia, and F. Martin, "Artificial left-handed transmission lines for small size microwave components: Application to power dividers," IEEE European Microwave Conference, Manchester (UK), pp. 1135-1138, Sept. 2006.

[12] J. Naqui, M. Durán-Sindreu, and F. Martín, "Novel sensors based on the symmetry properties of split ring resonators (SRRs)," Sensors, vol. 11, no. 8, pp. 7545-7553, 2011.

[13] M. Puentes, C. Weiss, M. Schüßler, and R. Jakoby, "Sensor array based on split ring resonators for analysis of organic tissues," IEEE MTT-S Int. Microwave Symp. Dig., Baltimore, MD, June 2011.

[14] I Arnedo, J. Illescas, M. Flores, M.A.G. Laso, F. Falcone, J. Bonache, J. García-García, F. Martín, J.A. Marcotegui, R. Marques, and M. Sorolla, 
"Forward and backward leaky wave radiation in split-ring-resonator based metamaterials," IET Microw. Ant. Propag., vol. 1, no. 1, pp. 6568, Feb. 2007.

[15] S. Eggermont and I. Huynen, "Influence of number of split rings on the leaky radiation of a metamaterial transmission line based on complementary split ring resonators," Microw. Opt. Techn. Lett., vol. 54 (4), pp. 867-875, Apr. 2012.

[16] G. Zamora, S. Zuffanelli, F. Paredes, F. Herraiz-Martinez, F. Martin, and J. Bonache, "Fundamental mode Leaky-Wave-Antenna (LWA) Using Slot Line and Split-Ring-Resonator (SRR) based metamaterials," IEEE Ant. Wireless Propag. Lett., vol. 12, pp. 1424-1427, Oct. 2013.

[17] K. Boratay Alici and E. Ozbay, "Electrically small split ring resonator antennas,” J. Appl. Phys., vol. 101, no. 8, p. 083104, 2007.

[18] J.J. Ma, X.Y. Cao, and T. Liu, "Design the size reduction patch antenna based on complementary split ring resonators," IEEE Int. Conf. Microw. Millim. Wave Tech., Chengdu, pp. 401-402, May 2010.

[19] R.W. Ziolkowski, P. Jin, and C.-C. Lin, "Metamaterial-Inspired Engineering of Antennas," Proc. of the IEEE, vol. 99, no. 10, pp. 17201731, Oct. 2011.

[20] F. J. Herraiz-Martinez, L. E. Garcia-Munoz, D. Gonzalez-Ovejero, V. Gonzalez-Posadas, and D. Segovia-Vargas, "Dual-frequency printed dipole loaded with split ring resonators," IEEE Ant. Wireless Propag. Lett., vol. 8, pp. 137-140, Jan. 2009.

[21] F. Paredes, G. Z. Gonzalez, F. J. Herraiz-Martinez, F. Martin, and J. Bonache, "Dual-band RFID tags based on folded dipole antennas loaded with spiral resonators," IEEE Int. Workshop on Antenna Technology, Tucson (AZ), pp. 136-139, Mar. 2012.

[22] M.-C. Tang and R.W. Ziolkowski, "A Study of Low-Profile, Broadside Radiation, Efficient, Electrically Small Antennas Based on Complementary Split Ring Resonators," IEEE Trans. Ant. Propag., vol. 61, no. 9, pp. 4419-4430, Sept. 2013.

[23] C. Xiaoyu, D. E. Senior, J.J. Whalen, and Y. K. Yoon, "Electrically small tunable split ring resonator antenna," IEEE Int. Symp. Ant. Propag., Toronto (ON), pp. 1-4, July 2010.

[24] J. Garcia-Garcia, F. Martin, J.D. Baena, R. Marques, and L. Jelinek, "On the resonances and polarizabilities of Split ring resonators," $J$. Appl. Phys., vol. 98, no. 3, p. 033103, Aug. 2005.

[25] M. Shamonin, E. Shamonina, V. Kalinin, and L. Solymar, "Resonant frequencies of a split-ring resonator: Analytical solutions and numerical simulations," Microw. Opt. Techn. Lett., vol. 44, no. 2, pp. 133-136, Jan. 2005.

[26] R. Marques, F. Medina, and R. Rafii-El-Idrissi, "Role of bi-anisotropy in negative permeability and left handed metamaterials," Phys. Rev. B, vol. 65, p. 144441, 2002.

[27] R. Marqués, F. Mesa, J. Martel, and F. Medina, “Comparative analysis of edge and broadside coupled split ring resonators for metamaterial design. Theory and experiment," IEEE Trans. Ant. Propag., vol. 51, pp. 2572-2582, 2003.

[28] B. Sauviac, C. R. Simovski, and S. A. Tretyakov. "Double split-ring resonators: Analytical modeling and numerical simulations," Electromagnetics, vol. 24, no. 5, pp. 317-338, 2004.

[29] M.D. Chiou and S.Y. Chen, "An electrically small planar antenna using complementary split-ring resonators," IEEE Int. Synp. Ant. Propag., Nagoys, pp. 1313-1316, 2012.

[30] J.S. McLean, "A re-examination of the fundamental limits on the radiation Q of electrically small antennas," IEEE Trans. Ant. Propag., vol. 44, no. 5, pp. 672-676, May 1996.

[31] J. D. Jackson, Classical Electrodynamics (3rd ed.), Wiley, 1998.

[32] S.J. Orfanidis, Electromagnetic Waves and Antennas, Rutgers University, 2000.

[33] D. M. Pozar, Microwave Engineering, (2nd ed.), New York: Wiley, 1998.

[34] P. N. Murgatroyd, "Calculation of proximity losses in multistranded conductor bunches," IEE Proceedings A (Physical Science, Measurement and Instrumentation, Management and Education), vol. 136, issue 3, pp. 115-120, May 1989.

[35] P. V. Nikitin, S.F. Lam, and K. V. S. Rao, "Low cost silver ink RFID tag antennas," IEEE APS International Symposium, vol. 2B, pp. 353356, July 2005.
[36] P. Pongpaibool, "A study of cost-effective conductive ink for inkjetprinted RFID application,” International Symposium on Antennas and Propagation (ISAP), pp. 1248-1251, 2012.

[37] A.D. Yaghjian and S.R. Best, "Impedance, bandwidth, and Q of antennas,” IEEE Trans. Ant. Propag., vol. 53, no. 4, pp. 1298-1324, Apr. 2005.

[38] L.J. Chu, "Physical limitations of omni-directional antennas," J. Appl. Phys., vol. 19, no. 12, pp. 1163-1175, Dec. 1948.

[39] W.A. Davis, T. Yang, E.D. Caswell, and W.L. Stutzman, "Fundamental limits on antenna size: a new limit," IET Microw. Ant. Propag., vol. 5, no. 11, pp. 1297-1302, Aug. 2011.

[40] M. Gustafsson, C. Sohl, and G. Kristensson, "Illustrations of New Physical Bounds on Linearly Polarized Antennas," IEEE Trans. Ant. Propag., vol. 57, no. 5, pp. 1319-1327, May 2009.

[41] K. Mohammadpour-Aghdam, R. Faraji-Dana, G.A.E. Vandenbosch, S. Radiom, and G.G.E. Gielen, "Physical bound on Q factor for planar antennas," Proc. 41st European Microwave Conf., pp. 250-252, Oct. 2011.

[42] Manufacturer datasheet.

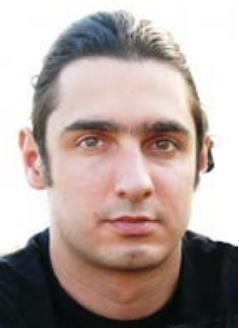

Simone Zuffanelli was born in Prato (Italy) in 1983. He received the Electronics Engineering Diploma in 2008 at the Università Degli Studi di Firenze. He obtained "Micro and Nanoelectronics Engineering" master's degree in 2011 at the Universitat Autònoma de Barcelona. He is currently working as a researcher in the field of metamaterial inspired antennas and RFID tags. His previous experiences include electronic design in the context of European projects "Persona" and "NOMS".

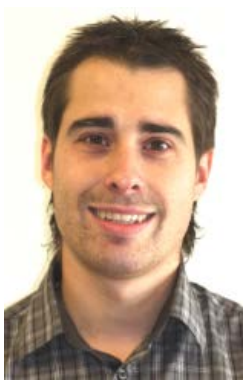

Gerard Zamora was born in 1984 in Barcelona (Spain). He received the Telecomunications Engineering Diploma, specialising in Electronics from the Universitat Autònoma de Barcelona in 2005. He obtained the Telecomunications Engineering degree in 2008 and the $\mathrm{PhD}$ degree from the same university in 2013. He is currently working as a researcher at the Universitat Autònoma de Barcelona. His research interests include antennas and microwave devices, the RFID technology and metamaterials.

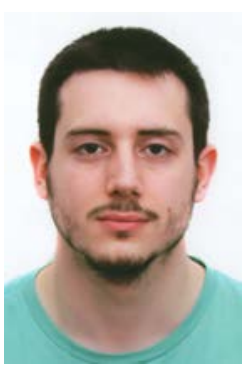

Pau Aguilà was born in Barcelona, Spain in 1989. He received the Telecommunications Engineering diploma (specializing in electronics) and the Telecommunications Engineering degree from the Universitat Autònoma de Barcelona in 2010 and 2012, respectively. He is currently working toward his $\mathrm{PhD}$ degree at the Universitat Autònoma de Barcelona in the field of metamaterials applied to microwave devices and RFID systems.

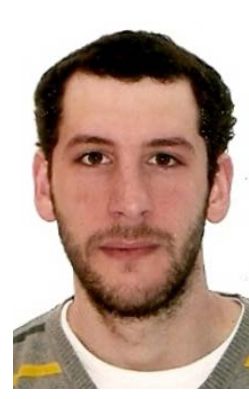

Ferran Paredes was born in Badalona (Barcelona), Spain in 1983. He received the Telecommunications Engineering Diploma (specializing in Electronics) and the Telecommunications Engineering degree from the Universitat Autònoma de Barcelona in 2004 and 2006, respectively and the $\mathrm{PhD}$ degree in Electronics Engineering from the same university in 2012. He was Assistant Professor from 2006 to 2008 at the Universitat Autònoma de Barcelona, where he is currently working as a Research Assistant. His research interests include metamaterial concepts, passive microwaves devices, antennas and RFID. 


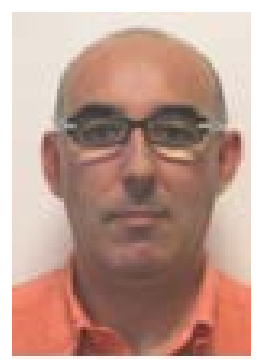

Ferran Martín (M’04-SM’08-F'12) was born in Barakaldo (Vizcaya), Spain in 1965. He received the B.S. Degree in Physics from the Universitat Autònoma de Barcelona (UAB) in 1988 and the $\mathrm{PhD}$ degree in 1992. From 1994 up to 2006 he was Associate Professor in Electronics at the Departament d’Enginyeria Electrònica (Universitat Autònoma de Barcelona), and since 2007 he is Full Professor of Electronics. In recent years, he has been involved in different research activities including modelling and simulation of electron devices for high frequency applications, millimeter wave and $\mathrm{THz}$ generation systems, and the application of electromagnetic bandgaps to microwave and millimeter wave circuits. He is now very active in the field of metamaterials and their application to the miniaturization and optimization of microwave circuits and antennas. He is the head of the Microwave Engineering, Metamaterials and Antennas Group (GEMMA Group) at UAB, and director of CIMITEC, a research Center on Metamaterials supported by TECNIO (Generalitat de Catalunya). He has organized several international events related to metamaterials, including Workshops at the IEEE International Microwave Symposium (years 2005 and 2007) and European Microwave Conference (2009), and the Fifth International Congress on Advanced Electromagnetic Materials in Microwaves and Optics (Metamaterials 2011), where he has acted as chair of the Local Organizing Committee. He has acted as Guest Editor for three Special Issues on Metamaterials in three International Journals. He has authored and co-authored over 450 technical conference, letter, journal papers and book chapters, he is co-author of the book on Metamaterials entitled Metamaterials with Negative Parameters: Theory, Design and Microwave Applications (John Wiley \& Sons Inc.), and he has generated 14 PhDs. Ferran Martín has filed several patents on metamaterials and has headed several Development Contracts.

Prof. Martín is a member of the IEEE Microwave Theory and Techniques Society (IEEE MTT-S). He is reviewer of the IEEE Transactions on Microwave Theory and Techniques and IEEE Microwave and Wireless Components Letters, among many other journals, and he serves as member of the Editorial Board of IET Microwaves, Antennas and Propagation and International Journal of RF and Microwave Computer-Aided Engineering. $\mathrm{He}$ is also a member of the Technical Committees of the European Microwave Conference (EuMC) and International Congress on Advanced Electromagnetic Materials in Microwaves and Optics (Metamaterials). Among his distinctions, Ferran Martín has received the 2006 Duran Farell Prize for Technological Research, he holds the Parc de Recerca UAB Santander Technology Transfer Chair, and he has been the recipient of two ICREA ACADEMIA Awards (calls 2008 and 2013).

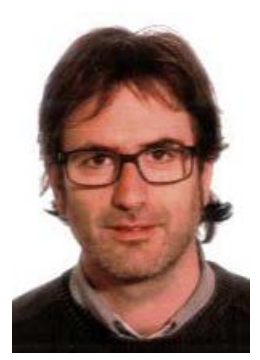

Jordi Bonache (S’05-M’07) was born in 1976 in Barcelona, Spain. He received the Physics and Electronics Engineering degrees and the Ph.D. degree in electronics engineering from the Universitat Autònoma de Barcelona, Barcelona, Spain, in 1999, 2001, and 2007, respectively. In 2000, he joined the "High Energy Physics Institute" of Barcelona (IFAE), where he was involved in the design and implementation of the control and monitoring system of the MAGIC telescope. In 2001, he joined the Department of Electronics Engineering of the Universitat Autònoma de Barcelona where he is currently Lecturer. From 2006 to 2009 he worked as executive manager of CIMITEC. Currently he is leading the research in RFID and antennas in CIMITEC. His research interests include active and passive microwave devices, metamaterials, antennas and RFID.

\footnotetext{
Cop. 2015 IEEE. Personal use of this material is permitted. Permissions from IEEE must be obtained for all other uses, in any current or future media, including reprinting/republishing this material for advertising or promotional purposes, creating new collective works, for resale or redistribution to servers or lists, or reuse of any copyrighted component of this work in other works.
} 